\title{
Minimally invasive plate osteosynthesis (MIPO) of periprosthetic femoral fractures with percutaneous cerclage wiring for fracture reduction: tips and technique
}

\author{
Jonna N. ${ }^{1}$, Manohar U. ${ }^{2 *}$
}

DOI: https://doi.org/10.17511/ijphr.2015.i1.03

\footnotetext{
1 Nagesh Jonna, Associate Professor, Department of Orthopedics, Rajiv Gandhi Institute of Medical Sciences, Kadapa, Andhra Pradesh, India.

2* U Rama Manohar, Assistant Professor, Department of Orthopedics, Rajiv Gandhi Institute of Medical Sciences, Kadapa, Andhra Prades, India.
}

Introduction: Periprosthetic femoral fractures (PPFs) associated at or near a well-fixed femoral prostheses (Vancouver type-B1) present a clinical challenge due to the quality of the bone stock and instability of the fracture. Combining closed reduction techniques with minimally invasive plate osteosynthesis (MIPO) or intramedullary nailing is a technically challenging procedure, especially when dealing with complex femoral fractures such as periprosthetic fractures. Cerclage wiring is a well known adjunct for fracture reduction and fixation. However, it is usually performed by open reduction, requiring wide surgical exposures, that results in soft tissue stripping. Materials and Methods: In our original study 18 patients with periprosthetic femoral shaft fracture (mean age, seventy-four years; range, forty-seven to eighty-four years) were treated with the described percutaneous cerclage wire and MIPO techniques. One patient died two months postsurgery, leaving seventeen patients who were followed for a mean of 13.2 months. Results and Discussion: Four patients sustained a spiral fracture pattern; three, an oblique fracture; and two, a wedge fracture. Closed reduction was successful in all but one case, and took a mean of 24.4 minutes (range, seven to forty-five minutes). The mean total operative time was 103 minutes (range, seventy-five to 140 minutes). Blood loss was $<100 \mathrm{ml}$ in all cases. All fractures united at a mean of eighteen weeks (range, sixteen to twenty weeks). No hardware failures were observed; one plate bent $10^{\circ}$, but the bending did not progress and the fracture healed uneventfully at sixteen weeks. Seven of the nine patients were able to return to their previous level of mobility.

Keywords: MIPO, Percutaneous Cerclage Wiring, Periprosthetic Femoral Fractures

Corresponding Author

U Rama Manohar, Assistant Professor, Department of Orthopedics, Rajiv Gandhi Institute of Medical Sciences, Kadapa, Andhra Prades, India.
How to Cite this Article

Jonna N, Manohar UR. Minimally invasive plate osteosynthesis (MIPO) of periprosthetic femoral fractures with percutaneous cerclage wiring for fracture reduction: tips and technique. Public Health Rev Int J Public Health Res. 2015;2(1):15-20. Available From

https://publichealth.medresearch.in/index.php/ijphr/ article/view/10

\section{To Browse}

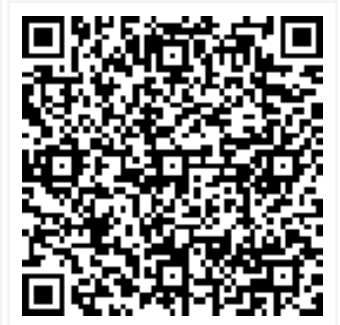

Manuscript Received 2015-03-10

Conflict of Interest No

Review Round 1
2015-03-16
Funding
Nil

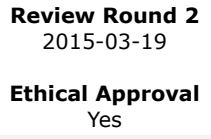

Review Round 3

Plagiarism X-checker $5 \%$
Accepted 2015-03-29

(C) 2015 by Nagesh Jonna, U Rama Manohar and Published by Siddharth Health Research and Social Welfare Society. This is an Open Access article licensed under a Creative Commons Attribution 4.0 International License https://creativecommons.org/licenses/by/4.0/ unported [CC BY 4.0]. 


\section{Introduction}

We describe percutaneous cerclage wiring and minimally invasive plate osteosynthesis (MIPO) for periprosthetic femoral shaft fractures. The evolution of MIPO in recent years has led to the development of new reduction tools to facilitate fracture reduction. This approach is meant to minimize additional trauma to the facture site, thus protecting vascularity and bone healing when applied in a minimally invasive fashion.

The deforming forces acting on periprosthetic femoral fractures, particularly femoral shaft fractures, make it challenging to obtain and maintain the reduction for plate application when using minimally invasive plate techniques. One viable option is the percutaneous application of cerclage, or circumferential, wires in combination with MIPO [1]. This has the advantage of minimizing the soft-tissue injury and dissection, preserving the fracture hematoma and blood supply to the bone fragments, and therefore promoting bone healing.

Although cerclage wires and cables are traditionally used in open surgical exposures, utilization of these devices as a reduction and fixation tool has been a long-standing tradition in orthopaedic surgery since Goetze first described their use for internal fixation in $1933[2,3]$. Cerclage fixation has often been employed for periprosthetic fractures, particularly around the hip, which provides extra fixation when a prosthesis occupies the femoral canal [4].

In general, cerclage wiring alone provides insufficient mechanical strength to maintain stable fixation; however, when used as an adjunct with an internal splint such as a plate, nail, or stem of a prosthesis, reliable stability can be achieved. From a biomechanical point of view, certain fractures even benefit from the "loose-lock stability" of cerclage wires or cables as a result of the relative instability promoting fracture healing by secondary fracture healing [5].

Recent evidence has refuted the historical argument that cerclage wiring may strangle the periosteal blood supply. In a cadaveric study using liquid contrast gelatin and three-dimensional computed tomography, one of us (T.A) and colleagues found no significant difference between the observed disruption of deep femoral arteries and perforating arteries, independent of the location of wiring or spacing between wire loops [6].
The technique with which the cerclage wiring is executed may have a greater impact than any potential effects from strangulation.

Open cerclage wiring requires extensive surgical dissection with a resultant disruption of the blood supply and evacuation of the hematoma. Regardless of whether an open or percutaneous technique is used, a major concern with cerclage wire application is soft-tissue interposition, especially of major neurological and vascular structures, as has been previously reported.

It is the purpose of this article to describe the percutaneous cerclage and MIPO technique for use in periprosthetic femoral shaft fractures in a stepwise fashion for safe execution [7]. A similar technique could be used for nonprosthetic spiral long-bone fractures. The procedure is performed in the following key steps [8].

Step 1: Preoperative Planning and Assessment of the Length, Alignment, and Rotation of the Extremity: A well-developed preoperative plan and assessment of the length, alignment, and rotation of the extremity are critical.

- A well-developed preoperative plan is essential. Carefully evaluate the fracture pattern to confirm the feasibility of cerclage wire reduction, which is most useful for approximating plate implants to bone and reducing spiral or long oblique fractures.

- With the patient supine on a radiolucent table, attempt to align the femoral fracture with any of the following manual reduction techniques: traction, a femoral distractor, and/or strategically placed bumps, depending on the fracture pattern. Verify the length, rotation, and alignment with an image intensifier.

- It is critical to assess the length, alignment, and rotation of the extremity. We prefer to do so with both lower extremities draped into the field so that the contralateral lower limb can be used as a clinical template for these assessments. Alternatively, to measure the length and alignment and rotation of the extremity using the C-arm:

- Compare the rotation of the contralateral extremity with that of the fractured side by assessing the position of the lesser trochanter at the hip and the fibula at the knee with the lower limb in a neutral position. These visuals can be compared side to side. 
- Place the cautery cord directly over the center of the femoral head proximally and the middle of the talus distally (while the extremity is in neutral rotation). The cord should run through the center of the knee for proper alignment of the extremity. Length can also be assessed with the cautery cord by comparing side-to-side measurements in this same way.

Step 2: Prepare the Tunnel : After making the appropriate incision, use the tunneling device anterior and posterior to the femur to create a softtissue tunnel.

- Make a 2 to $3-\mathrm{cm}$ longitudinal incision through the skin, subcutaneous tissue, and fascia over the proximal fracture fragment (i.e., proximal part of the diaphysis) using the image intensifier. Continue the deep dissection, splitting the iliotibial tract and vastus lateralis to the lateral aspect of the femur.

- Introduce the tunneling device (Synthes, Paoli, Pennsylvania) through the incision and perforate the intermuscular septum posteriorly just off the linea aspera to create a pathway for the cerclage wire passer. Use the tunneling device anterior and posterior to the femur to create a soft-tissue tunnel. As a general rule, the outermost cerclage wire should be at least $1 \mathrm{~cm}$ from the tip of the fracture line.

Step 3: Insert and Connect the Cerclage Passer: Insert the cerclage passer carefully by keeping it in close contact with the bone.

- The cerclage wire passer (Synthes) is a detachable two-piece instrument that disassembles for introduction and fastens after insertion of both pieces.

- Insert the soft-tissue trocars or flexible stylus into both tubular components of the modular cerclage passer forceps, so that soft tissue does not "catch" or enter the tube.

- Begin by inserting the posterior portion of the cerclage passer through the perforation in the intermuscular septum, dorsal to the femur and up around the medial edge of the femur past its anterior surface. Maintain the passer in close contact with the bone.

- With the posterior portion of the cerclage passer held steady, insert the anterior part of the forceps, applying slight pressure to maintain contact between the passer and the anterior surface of the femur.
It is extremely important to maintain the position of the posterior part of the forceps against the bone (lest it be pushed medially, risking entrapment of neurovascular structures) and bring the anterior portion to it. One passer tube follows the circumference of the femoral shaft dorsally; the other follows it ventrally.

- Connect the tubes by aligning the grooves on the handles of the cerclage passer and then clamping the handles at the proximal end with the swing clip. The handles should close easily without any force. If the tips of the passer are not meeting properly, the clip will not easily close over the cerclage passer handles. When reducing a proximal femoral fracture, perform this critical maneuver by lowering the handle of the passer so that the ends meet anterior to the femur off its medial edge. In cases of distal fracture, this technique should be used with great caution, but it can be done by raising the handle anteriorly, to prevent entrapment of the neurovascular structures on the medial side of the femur.

Step 4: Insert the Cerclage Wire and Remove the Wire Passer: The cerclage wire may be incrementally inserted according to the direction on the passer to prevent kinking.

- Verify accurate closure of the cerclage wire passer with the image intensifier and remove the soft-tissue trocars.

- Insert the wire or cable through the tube until it passes circumferentially around the femur in the direction of the arrow on the device. At times, a set of pliers is helpful to guide the wire or cable all of the way through. Next, unlock the swing clip and sequentially remove the cerclage passers. Before the tip of the wire comes out of the dpasser, use the pliers to grasp the end of it to prevent it from recoiling back into the passer.

- The use of this cerclage technique can help in two ways. First, it can aid in reducing or approximating the plate to the bone, or perhaps in reducing a spiral fracture of a long bone. In this way, it is not so much for fixation as it is for reduction. Therefore, the type of cerclage device, whether a cable or wire, does not matter, and the wire need not be cinched tightly [9]. Percutaneous crimping devices for cables have been developed to augment the cerclage system; however, percutaneously inserted wires can be easily cinched down with a conventional heavy needle-nosed pliers or needle driver. 
If cables or wires are used for the primary purpose of fixation, they should be tightened securely around the plate-bone interface and be deployed with complementary instrumentation for whichever cable system is chosen.

- If you are using more than one cerclage wire loop, loosely tighten the first loop with the wire holding forceps or pliers and then replicate this sequence for the other percutaneous cerclage wires. The length and rotation of the extremity must be checked prior to the next definitive step of wire tightening. We routinely use one wire loop for oblique fractures, two separate wire loops for spiral fractures (one at the proximal part of the fracture and one at the distal part), and two or three separate loops for comminuted wedge or spiral fractures.

- After all desired cerclage wire loops have been sequentially tightened, verify the length and rotation of the extremity and confirm that there is no gapping or overlap at the fracture site with the image intensifier. This can be performed as described above.

- Slide the percutaneous wire cutter (Synthes) through the small incision over the redundant wire until it comes into contact with the bone, and then pull back $1.5 \mathrm{~cm}$ and bend the wire onto the cortex of the femur before cutting the wire.

Step 5: Place and Fix the Submuscular Plate: Verify the alignment and length of the plate with intraoperative images and precontour the plate to fit the lateral aspect of the femur as necessary.

- The wires can be placed either underneath or over the plate. If you want to use the cerclage device as a reduction aid for the displaced fracture, then tighten it, reduce the fracture, and place the plate over it. Alternatively, the plate can be applied to the side of the bone in a submuscular fashion, and then the wire can be used around the plate to accomplish the same end. It may eb logistically more difficult to use the wire over the plate to achieve the reduction in some instances because you are working both on plate position and fracture reduction simultaneously.

- Mark the proximal and distal incisions for the plate on the skin on the basis of the fluoroscopic feedback. Then apply the plate in a bridging fashion to bypass the fracture site.
The length of the plate should allow for at least four locking screws proximal to the fracture site and three locking screws distal to the fracture site. More important than the number of screws on each side of the fracture is spreading the screws over a longer distance using a longer plate.

- All of the screws do not need to be locking screws [10]. Conventional cortical screws can be placed on either side of the fracture, before any locking screws are placed. The quality of conventional screw fixation will be proportionalto the quality of the bone density and, in the elderly, may not yield very much strength. However, such a screw sequence can be used to approximate the plate to the bone, which can be helpful before deploying locking screws.

- Make longitudinal incisions both proximal and distal to the fracture site on the basis of the templating with the image intensifier, and dissect down to the lateral cortex of the femur. First cut the iliotibial band longitudinally. Make these incisions through the iliotibial band generously to avoid struggling with plate insertion.

- Insert a tunneling instrument (Synthes) to prepare the tunnel for the plate, or the plate itself may be used to slide over the lateral aspect of the femur to create its own tunnel if it can be held and manipulated accurately. As long as the wires or cables are in intimate contact with the bone, they should not impede the passage of either the tunneling device or the implant.

- Insert the plate aligned with the lateral cortex and confirm the position with the image intensifier on both the anteroposterior and the lateral view [11].

- Temporarily fix the plate with Kirschner wires, before inserting screws. At this point, the plate should be closely approximated to the bone, with conventional screws, a collinear clamp, or some other approximation device. Verify the length relationship one last time with the image intensifier by assessing the fracture. Then insert locking screws proximally and distally for the definitive fixation. The proximal and distal incisions can be used to place screws, and these should be augmented with more screws placed percutaneously through stab incisions if desired on the basis of the preoperative planning. 
Periprosthetic screws may be preferable in the proximal fragment to avoid damage to the cement mantle. These screws are short and designed to be predrilled and to stop short of the prosthesis even if they do penetrate the cement mantle.

Step 6: Closure and Postoperative Rehabilitation: Encourage an immediate range of motion to aid in postoperative recovery.

- In some patients, a suction drain may be inserted but this is not required for all cases. Close the iliotibial band with a number-1 absorbable suture, followed by routine subcutaneous and skin closure as desired.

- Beginning on the second day postsurgery, encourage the patient to move the hip and knee. Generally, patients can be instructed to bear partial weight (20 to $40 \mathrm{lb}(10$ to $20 \mathrm{~kg}$ )) and walk with assistive devices for the first six weeks. Progressively increase the weightbearing status during weeks six to twelve and allow full weightbearing after three months of recovery.

\section{Results}

In our original study 18 patients with periprosthetic femoral shaft fracture (mean age, seventy-four years; range, forty-seven to eighty-four years) were treated with the described percutaneous cerclage wire and MIPO techniques. One patient died two months postsurgery, leaving nine patients who were followed for a mean of 13.2 months (range, twelve to eighteen months).

Four patients sustained a spiral fracture pattern; three, an oblique fracture; and two, a wedge fracture.Closed reduction was successful in all but one case, and took a mean of 24.4 minutes (range, seven to forty-five minutes). The mean total operative time was 103 minutes (range, seventyfive to 140 minutes). Blood loss was $<100 \mathrm{ml}$ in all cases. All fractures united at a mean of eighteen weeks (range, sixteen to twenty weeks).

No hardware failures were observed; one plate bent 100 , but the bending did not progress and the fracture healed uneventfully at sixteen weeks. Seven of the nine patients were able to return to their previous level of mobility. During the application of the percutaneous cerclage wires, there were no vascular or nerve injuries and none of the patients returned with any wound complications.

\section{Discussion and Conclusion}

It is important to understand that this is a technically demanding procedure with the potential for catastrophic complications. Entrapment of both the deep and the superficial femoral arteries has been previously reported with open cerclage wire techniques. Keeping the tips of the handles of the cerclage passing instrument more anterior to the mid-axis of the medial aspect of the femur in the proximal third and posterior to the mid-axis in the distal two-thirds should prevent these complications [12].

Also, it is extremely important to maintain the position of the posterior cerclage passer against the bone and bring the anterior portion down to meet it. This common misconception has been refuted in both animal and cadaver studies. Kirby and Wilson reported that they found no evidence of complete devascularization with the application of cerclage wires in six dogs and concluded that cerclage devices, even when flat and wide, do not restrict cortical vascularity when applied to intact bones.

More recently, one of us (T.A) and colleagues evaluated the extent of femoral vascular disruption in cadavers with liquid contrast gelatin injected into the femoral artery [13]. Using percutaneous cerclage wiring techniques, the authors discovered that there was in fact minimal disruption of the femoral blood supply and the associated perforators in the femur compensated to maintain femoral perfusion through anastomoses $[14,15]$.

On the basis of the results of these studies and multiple clinical investigations, it is believed that any historical ischemic complications arose from the extensive surgical dissection and soft-tissue stripping required with open techniques of fracture fixation or open cerclage wiring techniques.

\section{Reference}

01. Perren SM, Windhof M. Basic mechanobiology of bone healing, In- Babst R, Bavonratanavech S, Pesantes $R$, editors, Minimially invasive plate osteosynthesis. 2nd ed, New York- Thieme. 2012.

[Crossref]

02. Goetze O. (Subcutaneous wire suture in oblique tibial fractures). Arch Klin Chir. 1933;177;445-9. [Crossref] 
03. Xue H, Tu Y, Cai M, Yang A. Locking compression plate and cerclage band for type B1 periprosthetic femoral fractures preliminary results at average 30-month follow-up. J Arthro plasty. 2011 Apr;26(3)467;e1.

[Crossref]

04. Scott RD, Turner RH, Leitzes SM, Aufranc OE. Femoral fractures in conjunction with total hip replacement. J Bone Joint Surg Am. 1975 Jun;57(4)494-501.

[Crossref]

05. Ricci WM, Bolhofner BR, Loftus T, Cox C, Mitchell $\mathrm{S}$, Borrelli J Jr. Indirect reduction and plate fixation, without grafting, for periprosthetic femoral shaft fractures about a stable intramedullary implant. J Bone Joint Surg Am. 2005 Oct;87(10)2240-5.

[Crossref]

06. Perren SM, Fernandez Dell'Oca $A$, Lenz $M$, Windolf $M$. Cerclage, evolution and potential of a Cinderella technology- An overview with reference to periprosthetic fractures. Acta Chir Orthop Traumatol Cech. 2011;78(3)190-9.

[Crossref]

07. Old AB, McGrory BJ, White RR, Babikian GM. Fixation of Vancouver B1 peri-prosthetic fractures by broad metal plates without the application of strut allografts. J Bone Joint Surg Br. 2006 Nov;88(11)1425-9.

[Crossref]

08. Berend $\mathrm{Kr}$, Lombardi AV Jr, Mallory TH, Chonko DJ, Dodds KL, Adams JB. Cerclage wires or cables for the management of intraoperative fracture associated with a cementless, tapered femoral prosthesis- results at 2 to 16 years. J Arthroplasty. 2004 Oct;19(7)(Suppl 2)17-21. [Crossref]
09. Kirby BM, Wilson JW. Effect of circumferential bands on cortical vascularity and viability. J Orthop Res. 1991 Mar;9(2)174-9.

[Crossref]

10. Aleto T, Ritter MA, Berend ME. Case reportsuperficial femoral artery injury resulting from cerclage wiring during revision THA. Clin Orthop Relat Res. 2008 Mar;466(3)749-53.

[Crossref]

11. Apivatthakakul T, Phaliphot J, Leuvitoonvechkit S. Percutaneous cerclage wiring, does it disrupt femoral blood supply?- A cadaveric injection study. Injury. 2013 Feb;44(2)168-74.

[Crossref]

12. Farouk O, Krettek C, Miclau T, Schandelmaier $P$, Tscherne $\mathrm{H}$. The topography of the perforating vessels of the deep femoral artery. Clin Orthop Relat Res. 1999 Nov;(368)255-9.

[Crossref]

13. Mehta V, Finn HA. Femoral artery and vein injury after cerclage wiring of the femur- a case report. J Arthroplasty. 2005 Sep;20(6)811-4.

[Crossref]

14. Apivatthakakul T, Phornphutkul C, Bunmaprasert T, Sananpanich $K$, Fernandez Dell'Oca A. Percutaneous cerclage wiring and minimally invasive plate osteosynthesis (MIPO)- a percutaneous reduction technique in the treatment of Vancouver type B1 periprosthetic femoral shaft fractures. Arch Orthop Trauma Surg. 2012 Jun;132(6)813-22.

[Crossref]

15. Duncan CP, Masri BA. Fractures of the femur after hip replacement. Instr Course Lect. $1995 ; 44 ; 293-304$.

[Crossref] 